\title{
Comparison of beam profiles and wedge factors for physical and enhanced dynamic wedge
}

\begin{abstract}
The purpose of the work is to compare the beam profiles and wedge factors of physical and enhanced dynamic wedge. The study was conducted using the Varian clinac IX linear accelerator with different photon energies $6 \mathrm{MV}$ and $15 \mathrm{MV}$. The slab phantom arranged at a fixed depth (5cm depth) above the PTW seven29 ion chamber array. The physical and enhanced dynamic wedges different angles $\left(15^{\circ}, 30^{\circ}, 45^{\circ}\right.$ and $\left.60^{\circ}\right)$ were examined for the field sizes, $5 \times 5 \mathrm{~cm}^{2}, 10 \times 10 \mathrm{~cm}^{2}$ and $15 \times 15 \mathrm{~cm}^{2}$ by delivering 100 monitoring unit (MU). The same procedure is repeated using $0.6 \mathrm{cc}$ farmer type ionization chamber to find out the wedge factors. From the profiles obtained it was understood that as field size increases there seems only a slight difference between physical wedge (PW) and enhanced dynamic wedge (EDW) in the penumbral region of the profile. The percentage variation of measured dose between physical and enhanced dynamic wedge at shoulder region in beam profiles for the measured field sizes lies between 0.03 and 2.2. The standard deviation of measured dose at shoulder region, between different field sizes lies between 0.099 and 0.188 . Based on the results of the study, it can be concluded that the Seven29 2D ion chamber array and $0.6 \mathrm{cc}$ Farmer type ionisation chamber can be used effectively to obtain the dosimetric characteristics of both EDW and Physical wedge.
\end{abstract}

Keywords: physical wedge, enhanced dynamic wedge, beam profile, wedge factor
Volume 5 Issue I - 2018

\author{
Syam Kumar SA,' Aparna P,' Shelly Johny J,' \\ Geetha Satheeshan' \\ 'Department of Radiation Oncology, Malabar Cancer Centre, \\ India \\ ${ }^{2}$ Department of Physics, University of Calicut, India
}

Correspondence: Syam Kumar SA, Department of Radiation Oncology, Division of Radiation Physics, Malabar Cancer Centre, Thalassery, Kerala, India, Email syam.ppm@gmail.com

Received: December 29, 2017| Published: January 30, 2018
Abbreviations: MU, monitoring unit; PW, physical wedge; EDW, enhanced dynamic wedge; IMRT, intensity-modulated radiation therapy; PMMA, poly methyl metha acrylate; SSD, source to surface distance; $\mathrm{NC}$, nano coulomb

\section{Introduction}

The linear accelerator (linac) is a device that uses high-frequency electromagnetic waves to accelerate charged particles such as electrons to high energies through a linear tube. The high-energy electron beam can be used for treating superficial tumours, or it can be made to strike a target to produce x-rays for treating deep-seated tumours. In the majority of instances, the collimated beam emerging from an X-ray tube can be directly applied to the patient. In some circumstances, it is desirable to modify the spacial distribution of radiation within the patient by the insertion of material into the beam. This is called beam modifying devices.

The wedge is a beam modifier device. There are three types of wedges; Physical wedge, Dynamic wedge and Enhanced dynamic wedge. Physical wedge is usually made of a dense material, such as lead or steel. This is mounted on a transparent plastic tray which can be inserted in the beam at a specified distance from the source. This distance is arranged such that the wedge tray is always at a distance of at least $15 \mathrm{~cm}$ from the skin surface, so as to avoid destroying the skin-sparing effect of the megavoltage beam.

Another class of wedges are the dynamic wedges. These wedges are generated electronically by creating wedged beam profiles through dynamic motion of an independent jaw within the treatment beam. Dynamic wedges do not offer significant clinical advantages over the traditional metal wedges. Moreover, all wedges and compensators are now superseded by the new technology using dynamic multi-leaf collimators in conjunction with the intensitymodulated radiation therapy (IMRT).
Enhanced dynamic wedge is a clinical treatment that uses modality to deliver wedge-shaped photon dose distribution using computercontrolled dose delivery combined with upper jaw motion. Wedgeshaped isodoses are used for common clinical situations such as slope patient surfaces, regions of interesting beams and irregularly shaped tumor volumes. These isodose distributions can be achieved by many methods. ${ }^{1}$

Sendón Del Río et al. ${ }^{2}$ suggested a model that explored the observed behavior of virtual wedge factors and confirmed a strong dependence of virtual wedge factors on the dosimetric board. ${ }^{2}$ An explanation has been given on how the balance between different contributions is responsible for virtual wedge factors values. Dennis et al. ${ }^{3}$ suggested that it is necessary to know the dosimetric parameters for the treatment planning for enhancement of dynamic wedge. ${ }^{3}$

EDW technique differs from the physical (metal) wedge technique in that isodose profile is created by the sweeping action of the jaw from open to closed position while the beam is on. In this study a Seven29 detector array (PTW, Freiburg, Germany) was used to evaluate the beam profiles of physical and Enhanced dynamic wedge for different dimensions. Also to find the wedge factors for both types of wedges, $0.6 \mathrm{~cm}^{3}$ cylindrical ionisation chamber is used. A comparison study was also conducted between physical wedge and enhanced dynamic wedge.

\section{Materials and methods}

\section{PTW 2D array}

PTW Seven29 array detector (Freiburg Germany) is made up of 729 air vented cubic ionization chamber of $5 \mathrm{~mm} \times 5 \mathrm{~mm} \times 5 \mathrm{~mm}$ each with a center to center spacing of $1 \mathrm{~cm}$, is used for measuring dose generated in a plane by a radiation beam. Wall material is Graphite. The material is surrounded by Poly Methyl Metha Acrylate (PMMA). 
It is arranged uniformly in a $27 \times 27$ matrix. ${ }^{4}$ The advantage of using two dimensional array diode for QA is for the ability to perform absolute dose comparisons for hundreds of measurement positions using only a single beam delivery, as compared to the many multitudes of delivery repetitions necessary to perform absolute point measurements with a micro ionization - chamber.

\section{Slab phantom}

The density of slab phantom used in the study is close approximation to water. They provide a convenient and reliable alternative for photon and electron beam quality assurance. The material is flexible, and it does not break under impact. Thicknesses of slices are $0.1 \mathrm{~cm}$ to $7 \mathrm{~cm}$. The material can be easily machined and adapted to most ionization chambers.

\section{Farmer type ionisation chamber}

Farmer type ionisation chamber is a thimble chamber with graphite wall and graphite electrode for measuring high-energy photon and electron beam radiation. Thimble chamber can be used for in air and phantom measurements, to measure absolute dose in radiotherapy. Its waterproof design allows the chamber to be used in water or in solid state phantoms.

\section{D array and ionization chamber measurements}

The measurements were taken in the Varian Clinac iX linear accelerator using PTW 2D ion chamber array (Figure 1). This is done to obtain the beam profiles of physical wedge (PW) and enhanced dynamic wedge (EDW). $15^{\circ}, 30^{\circ}, 45^{\circ}$ and $60^{\circ}$ wedges IN orientation were examined. The measurements were taken for the energies $6 \mathrm{MV}$ and $15 \mathrm{MV}$. The measurement depth was $5 \mathrm{~cm}$. Slab phantoms with a fixed depth of $4.3 \mathrm{~cm}$ were arranged over the $2 \mathrm{D}$ array. Collimator and gantry angle was set to $0^{\circ}$. Source to surface distance (SSD) was $100 \mathrm{~cm} .100$ monitoring unit (MU) was delivered in each case. For each delivery, Verisoft Software provides a table of dose values which corresponds to each detector positions, isodose lines and isodose areas. The measurements were repeated for the field sizes $5 \times 5 \mathrm{~cm}^{2}$, $10 \times 10 \mathrm{~cm}^{2}$ and $15 \times 15 \mathrm{~cm}^{2}$.

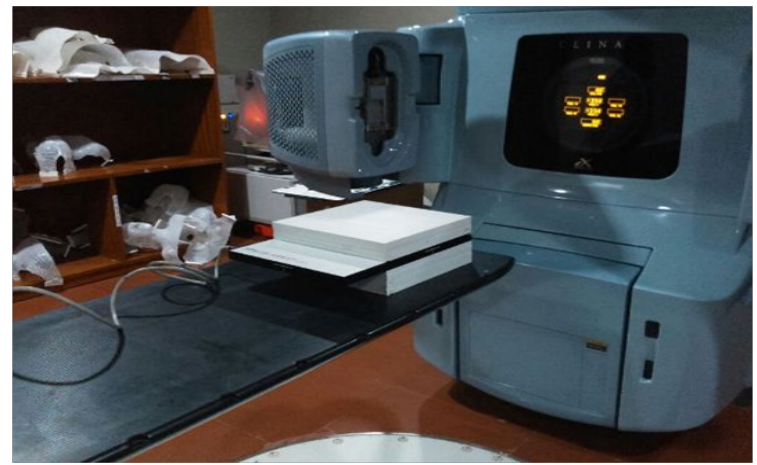

Figure I Measurement Set up in linac (Varian Clinac iX) where 2D array with solid water phantoms are arranged on the couch.

For wedge factor measurement, the same procedure was repeated with $0.6 \mathrm{~cm}^{3}$ cylindrical ionization chamber (Figure 2). The chamber was kept at $5 \mathrm{~cm}$ depth. The measurements were done for $6 \mathrm{MV}$ and 15 MV energies. The ionization chamber will be connected to the PTW Unidose electrometer for the measurements. Monitoring Unit of 100 MU will be delivered in each case. The electrometer readings were obtained in nano coulomb $(\mathrm{nC})$. To find the dose without wedge, an open field measurement were performed for the field sizes, $5 \times 5 \mathrm{~cm}^{2}$, $10 \times 10 \mathrm{~cm}^{2}$ and $15 \times 15 \mathrm{~cm}^{2}$ at $5 \mathrm{~cm}$ depth for $6 \mathrm{MV}$ and $15 \mathrm{MV}$ energies.

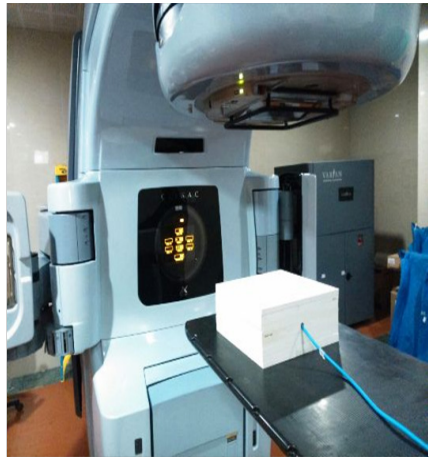

Figure 2 Measurement set up in linac (Varian Clinac iX) for the wedge factor determination.

\section{Results}

Beam profiles were reconstructed with the help of a spreadsheet program that was built in-house and that employed the data obtained from the 2D array measurements. Figure 3A-D gives the beam profiles of physical and enhanced dynamic wedge for $6 \mathrm{MV}$ energy. Figure $3 \mathrm{~A} \&$ Figure $3 \mathrm{~B}$ corresponds to wedge angles $30^{\circ}$ and $45^{\circ}$ respectively for $10 \mathrm{x} 10 \mathrm{~cm}^{2}$ field size. Figure $3 \mathrm{C} \&$ Figure $3 \mathrm{D}$ corresponds to beam profiles of same wedge angles with $15 \times 15 \mathrm{~cm}^{2}$ field size.
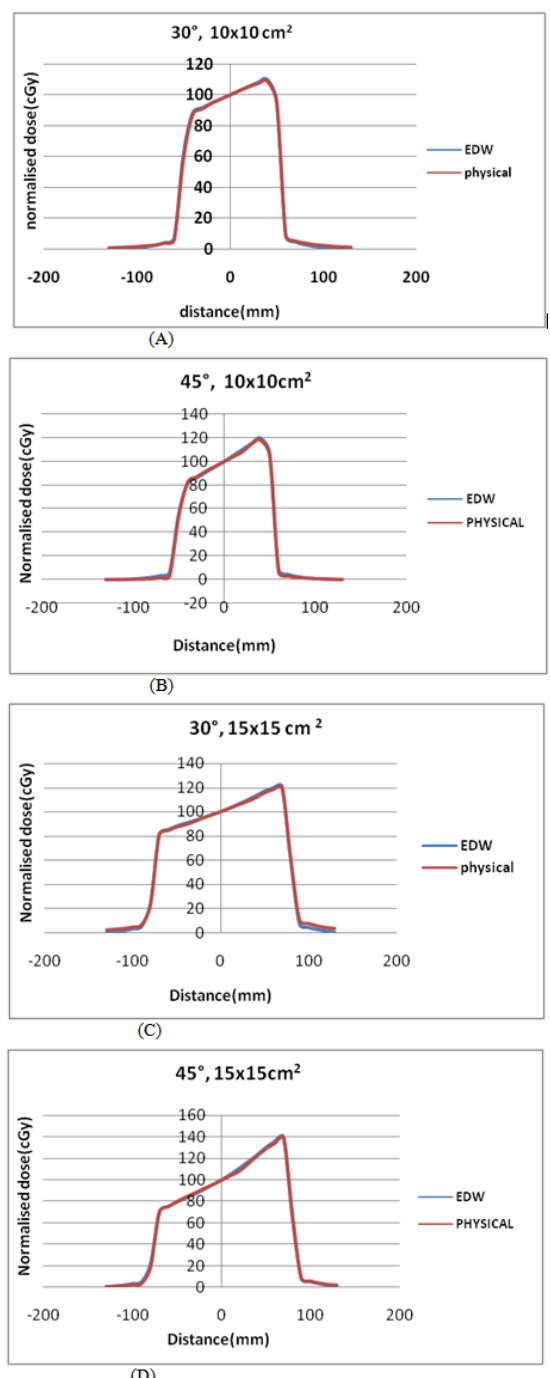

Figure 3 Beam profiles of physical and enhanced dynamic wedge for $6 \mathrm{MV}$ energy. 
Similarly, the Figure 4A-F gives the beam profiles of physical and enhanced dynamic wedge for $15 \mathrm{MV}$ energy. Figure $4 \mathrm{~A}, \mathrm{~B}$ corresponds to wedge angles $30^{\circ}$ and $45^{\circ}$ respectively for $10 \times 10 \mathrm{~cm}^{2}$ field size. Figure 4C \& Figure D corresponds to beam profiles of same wedge angles with $15 \times 15 \mathrm{~cm}^{2}$ field size.

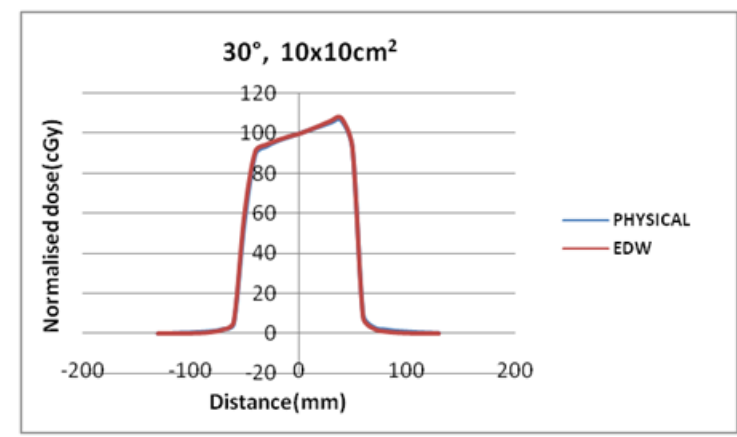

(A)

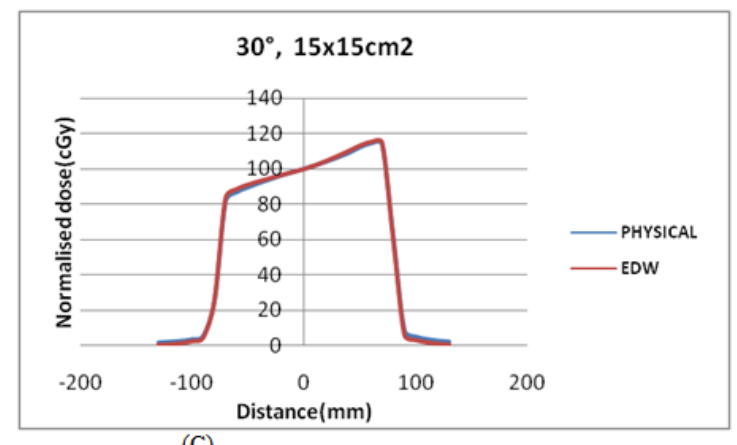

(C)
Table 1A \& Table 1B shows the percentage variation of measured dose between physical and enhanced dynamic wedge at shoulder region in beam profiles for $6 \mathrm{MV}$ and $15 \mathrm{MV}$ at $5 \mathrm{~cm}$ depth respectively. Table 2 shows the standard deviation of measured dose at shoulder region, between different field sizes $\left(5 \times 5,10 \times 10\right.$, and $\left.15 \times 15 \mathrm{~cm}^{2}\right)$.

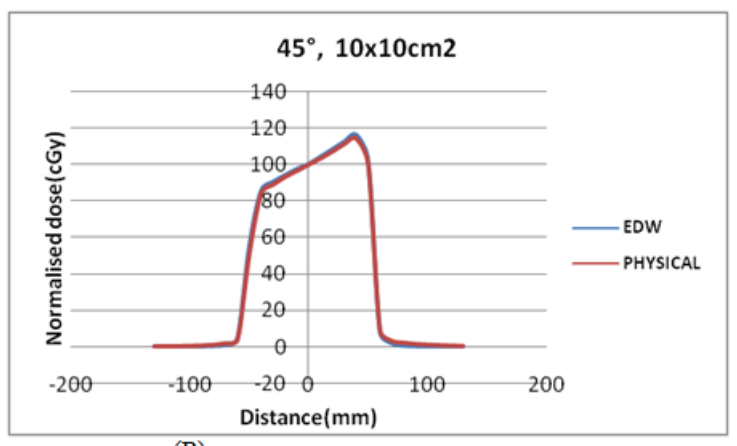

(B)

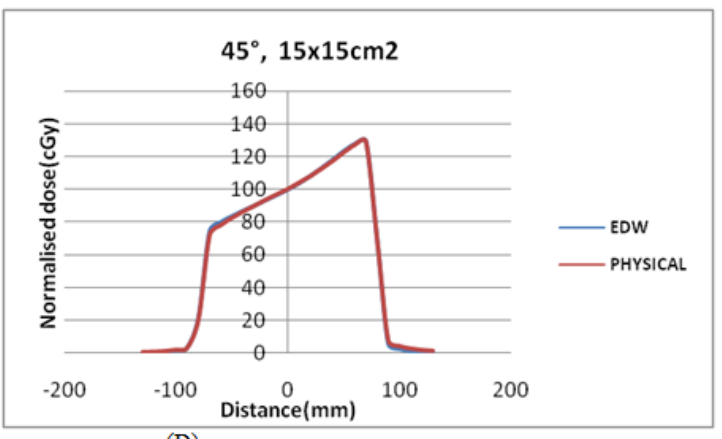

(D)

Figure 4 Beam profiles of physical and enhanced dynamic wedge for I5MV energy.

Table I Percentage variation of measured dose between physical and enhanced dynamic Wedge at shoulder region in beam profiles for; (A) 6MV energy at $5 \mathrm{~cm}$ depth; (B) I $5 \mathrm{MV}$ at $5 \mathrm{~cm}$ depth

\begin{tabular}{llll}
\hline Wedge angle (Degree) & \multicolumn{3}{l}{ Field sizes $\left(\mathrm{cm}^{2}\right)$} \\
\hline & $\mathbf{5 \times 5}$ & $10 \times 10$ & $\mathbf{1 5 \times 1 5}$ \\
\hline 15 & 0.085 & 0.52 & 0.81 \\
30 & 0.033 & 1.28 & 1.295 \\
45 & 1.63 & 3.81 & 6.7 \\
60 & 0.57 & 2.2 & 2.02 \\
\hline (A) & & &
\end{tabular}

\begin{tabular}{llll}
\hline Wedge angle (Degree) & \multicolumn{3}{c}{ Field sizes $\left(\mathbf{c m}^{2}\right)$} \\
\hline 15 & $\mathbf{5 X 5}$ & $10 \times 10$ & $\mathbf{1 5 X 1 5}$ \\
\hline 30 & 0.056 & 0.45 & 1.51 \\
45 & 0.106 & 1.075 & 1.008 \\
60 & 0.263 & 0.854 & 0.298 \\
\hline
\end{tabular}

(B)
Table 2 Standard deviation of measured dose at shoulder region, between different field sizes $(5 \times 5,10 \times 10$, and $15 \times 15)$

\begin{tabular}{lllll}
\hline \multirow{2}{*}{$\begin{array}{l}\text { Wedge angle (In } \\
\text { degree) }\end{array}$} & \multicolumn{2}{c}{ EDW } & & \multicolumn{2}{c}{ Physical wedge } \\
\cline { 2 - 5 } & $6 \mathrm{MV}$ & I5 MV & $6 \mathrm{MV}$ & I5 MV \\
\hline 15 & 0.1803 & 0.195 & 0.0096 & 0.1845 \\
30 & 0.172 & 0.1884 & 0.1497 & 0.1714 \\
45 & 0.1716 & 0.186 & 0.1303 & 0.147 \\
60 & 0.1675 & 0.1727 & 0.1492 & 0.1738 \\
\hline
\end{tabular}

\section{Discussion}

When profiles of physical and EDW obtained from 2D array measurement were compared, it was found that the profiles are in agreement with each other except some points in toe, heel and penumbral region, which is negligible.

From the Figure 3A-D \& 4A-D, it was understood that as field size increases, small difference between physical and EDW could see in the penumbral region of the profile. This may due to the increase in scattered radiation with the field size. Compared to EDW, physical wedges tend to increase in the penumbral region slightly. Also it can be due to the beam hardening effect in the case of physical wedges ${ }^{5}$. This effect is absent in the case of EDW.

A slight difference at toe and heel side between two profiles with field size is due to the fact that portion of physical wedge in the beam path is increased with increase in field size and hence a stronger beam 
hardening effect has occurred for PW. ${ }^{5}$ Beam hardening is increased with increase in thickness from toe to heal side for PW in the beam path.

As energy increases the difference in measured doses at toe, heel and penumbra region is reduced. This may due to the decrease in beam divergence with the energy. As wedge angle increases, the dose at the heel region increases while dose at toe and penumbral region decreases. In general, the wedge filter alters the beam quality by preferentially attenuating the lower energy photons (beam hardening) and, to a lesser extent by Compton scattering, which results in energy degradation (beam softening).

The field size dependence of wedge factors may be attributed due to the introduction of non-uniform scattering of photons in the presence of the wedges. In other words the dependence of wedge factor on field size is mainly due to change in the phantom and collimator scattering due to the presence of physical wedges. The scattered photon fluence increases with the irradiated wedge volume that increases with the field size. ${ }^{5}$ The non-linearity of field size may be because of the scattering is not from a point, rather from within the wedge volume.

EDW revealed strong dependency between field size and wedge factor. There seems a smooth and continuous decrease in the wedge factor with increasing field size. The decrease in wedge factor with field size is more apparent for thicker wedge, while for thinner wedges the decrease is comparatively less apparent but still significant (i.e. greater than two per cent) for all wedges at the studied energies. There is a great difference in the values of physical wedge factor (PWF) and enhanced dynamic wedge factor (EDWF). The variation in wedge factor of physical wedge with field size is less than $2 \%$. But in the case of EDW, the variation will be greater (up to $25 \%$ ). As energy increases, this variation seems to be lesser. The percentage variation of physical wedge between 6 and 15MV for the same field size and wedge angle is greater when compared to EDW (Table 3) (Table 4).

Table 3 The percentage variation of physical wedge between $6 \mathrm{MV}$ and I5 MV

\begin{tabular}{|c|c|c|}
\hline Field size $\left(\mathrm{cm}^{2}\right)$ & PW-30 $(\%)$ & PW- $45^{\circ}(\%)$ \\
\hline 5 & 9.8 & 7.29 \\
\hline 10 & II & 8 \\
\hline 15 & 11.8 & 8.6 \\
\hline
\end{tabular}

Table 4 The percentage variation of enhanced dynamic wedge between $6 \mathrm{MV}$ and I5MV

\begin{tabular}{|c|c|c|}
\hline Field size $\left(\mathrm{cm}^{2}\right)$ & EDW-30 $0^{\circ}(\%)$ & EDW- $45^{\circ}(\%)$ \\
\hline 5 & 1.5 & 1.8 \\
\hline 10 & 3.6 & 5.9 \\
\hline 15 & 8 & 10.2 \\
\hline
\end{tabular}

The decrease in EDWF with field size can be explained from the fact that in general the exact progression of dose rate and jaw speed, as well as the total dose delivered as an open field depends on wedge angle, field size and monitor units. Since EDW uses variable dose rate and the jaw speed, which contribute high doses to the "toe" side of the wedge field with increase in field size and consequently the central axis accumulated dose decreases which causes a decrease in the central axis wedge factor. ${ }^{5}$ Different studies have been published on the field-size dependence, depth dependence, and off-axis dependence of physical and non-physical wedges..$^{5-15}$

From the Table 1A \& Table 1B it was cleared that the percentage variation of measured dose between physical and enhanced dynamic Wedge at shoulder region in beam profiles increases with energy and field sizes. But this increase is less significant. From Table 2, Standard deviation of measured dose at shoulder region, between different field sizes $(5 \times 5,10 \times 10$, and $15 \times 15)$ is less and within tolerance.

\section{Conclusion}

Based on the results of our study, it can be concluded that the Seven29 2D ion chamber array and 0.6cc Farmer-type ionisation chamber can be used effectively to obtain the dosimetric characteristics of both EDW and Physical wedge. With the in-house built program using spread sheet, the wedge profiles and wedge factors can be reconstructed from the $2 \mathrm{D}$ array and ionisation chamber measurements, which is easy and accurate.

\section{Acknowledgements}

None.

\section{Conflict of interest}

Author declares that there is no conflict of interest.

\section{References}

1. Muhammad Isa, Khalid Iqbal, Afzal M, et al. JRMO. 2009;19:33-38.

2. Sendon Del Rio JR, Martinez CO, Garcia MS, et al. Study and evaluation of the Siemens virtual wedge factor: dosimetric monitor system and variable field effects. Phys Med Biol. 2008;53(5):1313-1323.

3. Leavitt DD, Lee WL, Gaffney DK, et al. Dosimetric parameters of Enhanced Dynamic Wedge for treatment planning and verification. Me dical Dosimetry. 1997;22(3):177-183.

4. Mohammad Hussein, Elizabeth J Adams, Thomas J Jordan, et al. A critical evaluation of the PTW 2D-ARRAY seven29 and OCTAVIUS II phantom for IMRT and VMAT verification. J Appl Clin Med Phys. 2013;14(6):4460.

5. Ahmad M, Hussain A, Muhammad W, et al. Studying wedge factors and beam profiles for physical and enhanced dynamic wedges. J Med Phys. 2009;35(1):33-41.

6. Salk J, Blank P, Machold U, et al. Physical aspects in the clinical implementation of the Enhanced Dynamic Wedge (EDW). p. 1-48.

7. Gibbons JP. Calculation of enhanced dynamic wedge factors for symmetric and asymmetric photon fields. Med Phys. 1998;25(8):1411-1418.

8. Sehti A, Leybovich LB, Dogan N, et al. Elimination of field sizedependence of enhanced dynamic wedge factors. Phys Med Biol. 2000;45(11):3359-3365.

9. Leavitt DD, Lee WL, Gaffney DK, et al. Dosimetric parameters of enhanced dynamic wedge for treatment planning and verification. Med Dosim. 1997;22(3):177-183

10. McCullough EC, Gortney J, Blackwell RC. A depth dependence determination of wedge transmission factor for 4-10 MV photon beams. Med Phys. 1998;15(4):621-623.

11. Popescu A, Lai K, Singer K, et al. Wedge factor dependence with depth, field size, and nominal distances: a general computational rule. Med Phys. 1999;26(4):541-549. 
12. Tailor RC, Followill DS, Hanson WF. A first order approximation of field-size and depth dependence of wedge transmission. Med Phys. 1998;25(2):241-244.

13. Liu C, Li Z, Palta JR. Characterizing output for the Varian enhanced dynamic wedge field. Med Phys. 1998;25(1):64-70.
14. Zhu XR, Gillin MT, Jursinic PA, et al. Comparison of dosimetric characteristics of siemens virtual and physical wedges. Medical Physics. 2000;27(10):2267-2277.

15. Palta JR, Daftari I, Suntharalingam N. Field size dependence of wedge factors. Med Phys. 1998;15(4):624-626. 